\title{
SIGNED STRONG ROMAN DOMINATION IN GRAPHS
}

\author{
LEILA ASGHARSHARGHI, RANA KHOEILAR AND SEYED MAHMOUD SHEIKHOLESLAMI
}

\begin{abstract}
Let $G=(V, E)$ be a finite and simple graph of order $n$ and maximum degree $\Delta$. A signed strong Roman dominating function (abbreviated SStRDF) on a graph $G$ is a function $f: V \rightarrow\left\{-1,1,2, \ldots,\left\lceil\frac{\Delta}{2}\right\rceil+1\right\}$ satisfying the conditions that (i) for every vertex $v$ of $G, \sum_{u \in N[v]} f(u) \geq 1$, where $N[v]$ is the closed neighborhood of $v$ and (ii) every vertex $v$ for which $f(v)=-1$ is adjacent to at least one vertex $u$ for which $f(u) \geq 1+\left\lceil\frac{1}{2}\left|N(u) \cap V_{-1}\right|\right\rceil$, where $V_{-1}=\{v \in V \mid f(v)=-1\}$. The minimum of the values $\sum_{v \in V} f(v)$, taken over all signed strong Roman dominating functions $f$ of $G$, is called the signed strong Roman domination number of $G$ and is denoted by $\gamma_{s s R}(G)$. In this paper we initiate the study of the signed strong Roman domination in graphs and present some (sharp) bounds for this parameter.
\end{abstract}

\section{Introduction}

In this paper, $G$ is a simple graph with vertex set $V=V(G)$ and edge set $E=E(G)$. For every vertex $v \in V$, the open neighborhood $N_{G}(\nu)=N(v)$ is the set $\{u \in V \mid u v \in E\}$ and the closed neighborhood of $v$ is the set $N[v]=N(v) \cup\{v\}$. The degree of a vertex $v \in V$ is $d_{G}(v)=$ $d(\nu)=|N(v)|$. The minimum and maximum degree of a graph $G$ are denoted by $\delta=\delta(G)$ and $\Delta=\Delta(G)$, respectively. The complement of $G$ is denoted by $\bar{G}$. We write $K_{n}$ for the complete graph, $C_{n}$ for a cycle, $P_{n}$ for a path of order $n$ and $K_{1, r}$ for a star of order $r+1$. For $r, s \geq 1$, a double star $S(r, s)$ is a tree with exactly two vertices that are not leaves, with one adjacent to $r$ leaves and the other to $s$ leaves.

A subset $S$ of vertices of $G$ is a dominating set if $N[S]=V$. The domination number $\gamma(G)$ is the minimum cardinality of a dominating set of $G$. A dominating set of minimum cardinality of $G$ is called a $\gamma(G)$-set. A signed Roman dominating function (abbreviated SRDF) on $G$ is defined as a function $f: V(G) \longrightarrow\{-1,1,2\}$ such that $f(N[\nu])=\sum_{x \in N[v]} f(x) \geq 1$ for every $v \in V(G)$ and every vertex $u$ for which $f(u)=-1$ is adjacent to at least one vertex $v$ for which $f(v)=2$. The weight of an SRDF $f$ on a graph $G$ is $\omega(f)=\sum_{\nu \in V(G)} f(\nu)$. The signed Roman

Received June 12, 2016, accepted February 24, 2017.

2010 Mathematics Subject Classification. 05C69.

Key words and phrases. Signed strong Roman dominating function, signed strong Roman domination number.

Corresponding author: L. Asgharsharghi. 
domination number $\gamma_{s R}(G)$ of $G$ is the minimum weight of an SRDF on $G$. The signed Roman domination number was introduced by Ahangar et al. [1] and has been studied by several authors (for example [7]).

The defensive strategy of signed Roman domination is based in the fact that every place in which there is established a Roman legion (a label 1) is able to protect itself under external attacks; and that every place with an auxiliary troop (a label -1) must have at least a stronger neighbor (a label 2). In that way, if an unsecured place (a label -1) is attacked, then a stronger neighbor could send one of its two legions in order to defend the weak neighbor vertex (label -1) from the attack. If several simultaneous attacks to weak places are developed, then the only stronger place will be not able to defend its neighbors efficiently. With this motivation in mind, we introduce the concept of signed strong Roman dominating function as follows. For our purposes, we consider that a strong place should be able to defend itself and, at least half of its weak neighbors.

In graph theoretic terms, we define a signed strong Roman dominating function (SStRDF) on a graph $G=(V, E)$ to be a function $f: V \rightarrow\left\{-1,1,2, \ldots,\left\lceil\frac{\Delta}{2}\right\rceil+1\right\}$ satisfying the conditions that the sum of the values assigned to a vertex and its neighbors is at least 1 for every vertex and every vertex $v$ for which $f(v)=-1$ is adjacent to at least one vertex $u$ for which $f(u) \geq 1+\left\lceil\frac{1}{2}\left|N(u) \cap V_{-1}\right|\right\rceil$, where $V_{-1}=\{v \in V \mid f(v)=-1\}$. The signed strong Roman domination number, denoted $\gamma_{s s R}(G)$, is the minimum weight of an SStRDF in $G$; that is, $\gamma_{s s R}(G)=$ $\min \{\omega(f) \mid f$ is an SStRDF in $G\}$. An SStRDF of weight $\gamma_{s s R}(G)$ is called a $\gamma_{s s R}(G)$-function.

A signed strong Roman dominating function $f: V \rightarrow\left\{-1,1,2, \ldots,\left\lceil\frac{\Delta}{2}\right\rceil+1\right\}$ can be represented by the ordered partition $\left(V_{-1}, V_{1}, \ldots, V_{\left\lceil\frac{\Delta}{2}\right\rceil+1}\right)$ (or $\left(V_{-1}^{f}, V_{1}^{f}, \ldots, V_{\left\lceil\frac{\Delta}{2}\right\rceil+1}^{f}\right)$ to refer to $f$ ) of $V$, where $V_{i}=\{v \in V \mid f(v)=i\}$ for $i=-1,1, \ldots,\left\lceil\frac{\Delta}{2}\right\rceil+1$. If $G_{1}, G_{2}, \ldots, G_{s}$ are the components of $G$, then

$$
\gamma_{s s R}(G)=\sum_{i=1}^{s} \gamma_{s s R}\left(G_{i}\right) .
$$

In this paper, we initiate the study of the signed strong Roman domination in graphs and present some (sharp) bounds for this parameter. In addition, we determine the signed strong Roman domination number of some classes of graphs.

We make use of the following observations.

Proposition A ([1]). Let $G$ be a graph of order $n$. Then $\gamma_{s R}(G) \leq n$, with equality if and only if $G=\overline{K_{n}}$.

Observation 1. For any connected graph $G$ with $\Delta(G) \leq 2, \gamma_{s s R}(G)=\gamma_{s R}(G)$.

Proposition B ([1]). For $n \geq 3, \gamma_{s R}\left(C_{n}\right)=\left\lceil\frac{2 n}{3}\right\rceil$ and $\gamma_{s R}\left(P_{n}\right)=\left\lfloor\frac{2 n}{3}\right\rfloor$.

Corollary 2. For $n \geq 3, \gamma_{s s R}\left(C_{n}\right)=\left\lceil\frac{2 n}{3}\right\rceil$ and $\gamma_{s s R}\left(P_{n}\right)=\left\lfloor\frac{2 n}{3}\right\rfloor$. 
Based on (1) and Observation 1, from now on, we focus mainly on connected graphs with maximum degree $\Delta \geq 3$.

Proposition 3. For any integer $k \geq 1$, there exists a connected graph $G$ such that $\gamma_{s s R}(G)=-k$.

Proof. Let $v$ be the center of the star $K_{1,5 k+5}$ and let $G$ be a wounded spider obtained from $K_{1,5 k+5}$ by subdividing $k+1$ edges. Define $f: V \rightarrow\left\{-1,1,2, \ldots,\left\lceil\frac{\Delta}{2}\right\rceil+1\right\}$ by $f(x)=-1$ if $x$ is a leaf, $f(v)=2 k+3$ and $f(x)=2$ otherwise. Obviously, $f$ is an SStRDF on $G$ of weight $-k$ and so $\gamma_{s s R}(G) \leq-k$. On the other hand, for any $\gamma_{s s R}(G)$-function $g$, we have $g(N[\nu]) \geq 1$ and so

$$
\gamma_{s s R}(G) \geq g(N[v])-(k+1) \geq 1-(k+1)=-k .
$$

This implies that $\gamma_{s s R}(G)=-k$ and the proof is complete.

Proposition 4. For $n \geq 4, \gamma_{s s R}\left(K_{n}\right)=1$.

Proof. Let $V\left(K_{n}\right)=\left\{v_{1}, \ldots, v_{n}\right\}$ be the vertex set of $K_{n}$ and let $f$ be a $\gamma_{s s R}\left(K_{n}\right)$-function. Clearly $\gamma_{s s R}\left(K_{n}\right)=f\left(N\left[v_{n}\right]\right) \geq 1$. If $n$ and $\left\lceil\frac{n-1}{2}\right\rceil$ have different parity, then the function $f: V\left(K_{n}\right) \rightarrow$ $\left\{-1,1,2, \ldots,\left\lceil\frac{n-1}{2}\right\rceil+1\right\}$ defined by $f\left(v_{n}\right)=\left\lceil\frac{n-1}{2}\right\rceil+1, f\left(v_{i}\right)=-1$ for $1 \leq i \leq\left\lceil\frac{n-1}{2}\right\rceil, f\left(v_{j}\right)=(-1)^{j}$ for $\left\lceil\frac{n-1}{2}\right\rceil+1 \leq j \leq n-1$, is a signed strong Roman dominating function on $K_{n}$ of weight 1 . Let $n$ and $\left\lceil\frac{n-1}{2}\right\rceil$ have the same parity. Then the function $f$ defined by $f\left(v_{n}\right)=\left\lceil\frac{n-1}{2}\right\rceil, f\left(v_{i}\right)=-1$ for $1 \leq i \leq\left\lceil\frac{n-1}{2}\right\rceil, f\left(v_{j}\right)=(-1)^{j}$ for $\left\lceil\frac{n-1}{2}\right\rceil+1 \leq j \leq n-2$ and $f\left(v_{n-1}\right)=1$, is a signed strong Roman dominating function on $K_{n}$ of weight 1 . Thus $\gamma_{s s R}\left(K_{n}\right)=1$.

\section{Bounds on the signed strong Roman domination number}

In this section, we establish some sharp bounds on the signed strong Roman domination number.

Proposition 5. If $G$ is a connected graph of order $n$ with maximum degree $\Delta$, then

$$
\gamma_{s s R}(G) \geq 2+\Delta-n
$$

This bound is sharp for complete graphs $K_{n}(n \geq 4)$.

Proof. Let $f$ be a $\gamma_{s s R}(G)$-function and $v$ be a vertex of degree $\Delta$. Since $f(N[v]) \geq 1$, we have

$$
\gamma_{s s R}(G)=\omega(f) \geq 1-(n-\Delta-1)=2+\Delta-n \text {. }
$$

Proposition 6. Let $G$ be a connected graph of order $n$. Then the following statements hold.

(a) $\gamma_{s s R}(G) \leq n$, with equality if and only if $G=\overline{K_{n}}$.

(b) $\gamma_{s s R}(G) \geq 2 \gamma(G)-n$, with equality if and only if $G=\bar{K}_{n}$. 
Proof. (a) Clearly, assigning a weight of 1 to every vertex of $G$ produces an SStRDF of $G$ of weight $n$ which implies that $\gamma_{s s R}(G) \leq n$. If $G=\overline{K_{n}}$, then it follows from Proposition A and Observation 1 that $\gamma_{s s R}(G)=n$. Henceforth, we assume that $G$ is not empty. Let $G_{1}$ be a component of $G$ of order at least two, $u$ a vertex of minimum degree in $G_{1}$, and $v$ be a neighbor of $u$. Clearly the function $f: V(G) \rightarrow\left\{-1,1,2, \ldots,\left\lceil\frac{\Delta}{2}\right\rceil+1\right\}$ defined by $f(u)=-1, f(v)=2$ and $f(x)=1$ otherwise, is an SStRDF of $G$ of weight $n-1$ and so $\gamma_{s s R}(G) \leq n-1$.

(b) Let $f$ be a $\gamma_{s s R}(G)$-function. If $V_{i}=\varnothing$, for each $2 \leq i \leq\left\lceil\frac{\Delta}{2}\right\rceil+1$, then $V=V_{1}$, and we have $\gamma_{s s R}(G)=\omega(f)=n$. But then by (a), $G=\bar{K}_{n}$, and so $\gamma(G)=n$, implying that $\gamma_{s s R}(G)=2 \gamma(G)-$ $n$. Hence, we may assume that $\left|V_{i}\right| \geq 1$ for some $2 \leq i \leq\left\lceil\frac{\Delta}{2}\right\rceil+1$. Since $\left|V_{-1}\right|=n-\sum_{i=1}^{\left\lceil\frac{\Delta}{2}\right\rceil+1}\left|V_{i}\right|$ and $\cup_{i=1}^{\left\lceil\frac{\Delta}{2}\right\rceil+1} V_{i}$ is a dominating set for $G$, we have

$$
\begin{aligned}
\gamma_{s s R}(G) & =\sum_{i=1}^{\left\lceil\frac{\Delta}{2}\right\rceil+1} i\left|V_{i}\right|-\left|V_{-1}\right| \\
& =\sum_{i=1}^{\left\lceil\frac{\Delta}{2}\right\rceil+1}(i+1)\left|V_{i}\right|-n \\
& >2\left(\sum_{i=1}^{\left\lceil\frac{\Delta}{2}\right\rceil+1}\left|V_{i}\right|\right)-n \\
& \geq 2 \gamma(G)-n .
\end{aligned}
$$

This completes the proof.

Proposition 7. If $G$ is a connected graph of order $n$ with minimum degree $\delta(G)$, then $\gamma_{s s R}(G) \leq$ $n-\delta(G)+1+\left\lceil\frac{\delta(G)}{4}\right\rceil$.

Proof. The result is immediate for $\delta(G)=1$. Assume that $\delta(G) \geq 2$ and $\delta(G) \equiv r(\bmod 2)$. Let $v$ be a vertex of degree $\delta(G)$ and $N(v)=\left\{u_{1}, u_{2}, \ldots, u_{\delta(G)}\right\}$. Define the function $g: V \rightarrow$ $\left\{-1,1,2, \ldots,\left\lceil\frac{\Delta}{2}\right\rceil+1\right\}$ by $g\left(u_{i}\right)=(-1)^{i}$ for $1 \leq i \leq \delta(G)-r, g(v)=\left\lceil\frac{\delta(G)-r}{4}\right\rceil+1$ and $g(x)=1$ otherwise. It is easy to verify that $g$ is an SStRDF on $G$ of weight at most $n-\delta(G)+\left\lceil\frac{\delta(G)}{4}\right\rceil+1$ and hence

$$
\gamma_{s s R}(G) \leq n-\delta(G)+\left\lceil\frac{\delta(G)}{4}\right\rceil+1 .
$$

A set $S \subseteq V$ is a 2-packing of a graph $G$ if $N[u] \cap N[\nu]=\varnothing$ for any two distinct vertices $u, v \in S$. The 2-packing number $\rho(G)$ of $G$ is defined by

$$
\rho(G)=\max \{|S|: S \text { is a 2-packing of } G\} .
$$

Proposition 8. If $G$ is a graph of order $n$ with $\delta \geq 1$, then $\gamma_{s s R}(G) \geq \rho(G)(\delta+2)-n$. This bound is sharp for $S(2,2)$. 
Proof. Let $\left\{v_{1}, v_{2}, \ldots, v_{\rho(G)}\right\}$ be a 2-packing of $G$, and let $f$ be a $\gamma_{s s R}(G)$-function. Suppose that $A=\cup_{i=1}^{\rho(G)} N\left[v_{i}\right]$. Since $\left\{v_{1}, v_{2}, \ldots, v_{\rho(G)}\right\}$ is a 2-packing, we have

$$
|A|=\sum_{i=1}^{\rho(G)}\left(d_{G}\left(v_{i}\right)+1\right) \geq(\delta+1) \rho(G) .
$$

This implies that

$$
\begin{aligned}
\gamma_{s s R}(G) & =\sum_{x \in V} f(x)=\sum_{i=1}^{\rho(G)} f\left(N\left[v_{i}\right]\right)+\sum_{x \in V-A} f(x) \\
& \geq \rho(G)+\sum_{x \in V-A} f(x) \geq \rho(G)-n+|A| \\
& \geq \rho(G)-n+(\delta+1) \rho(G)=(\delta+2) \rho(G)-n .
\end{aligned}
$$

Lemma 9. Let $G$ be a connected graph of order $n \geq 4$. If $f$ is an SStRDF of $G$, then $\left|V_{-1}^{f}\right|<n-1$.

Proof. Let $f$ be an SStRDF of $G$ and assume to the contrary that $\left|V_{-1}^{f}\right|=n-1$. Then $1=$ $\sum_{i=2}^{\left\lceil\frac{\Delta}{2}\right\rceil+1}\left|V_{i}^{f}\right|$ and $\left|V_{1}^{f}\right|=0$. Let $\cup_{i=2}^{\left\lceil\frac{\Delta}{2}\right\rceil+1} V_{i}^{f}=\{x\}$. Since $f(N[y]) \geq 1$ for each $y \in V(G)$, we must have $x \in N(y)$. Thus $d_{G}(x)=n-1$. It follows from $f(x) \leq\left\lceil\frac{n-1}{2}\right\rceil+1$ that $f(N[x]) \leq 0$ which is a contradiction.

Proposition 10. Let $G$ be a connected graph of order $n \geq 4$. Then

$$
\gamma_{s s R}(G) \geq 3-\left\lfloor\frac{n}{2}\right\rfloor .
$$

Moreover, this bound is sharp for $S(2,4)$.

Proof. Let $f$ be a $\gamma_{s s R}(G)$-function and let $W=\bigcup_{i=2}^{\left[\frac{\Delta}{2}\right]+1} V_{i}$. Define $V_{-1}^{1}=\left\{v \in V_{-1}:|N(v) \cap W|=\right.$ $1\}$ and $V_{-1}^{2}=\left\{v \in V_{-1}:|N(v) \cap W| \geq 2\right\}$. Since $n=\left|V_{-1}\right|+\left|V_{1}\right|+|W|$, we have

$$
\begin{aligned}
\gamma_{s s R}(G) & =\left|V_{1}\right|-\left|V_{-1}\right|+\sum_{v \in W} f(v) \\
& \geq\left|V_{1}\right|-\left|V_{-1}\right|+|W|+\sum_{x \in W} \frac{1}{2}\left|V_{-1} \cap N(x)\right| \\
& =n-2\left|V_{-1}\right|+\sum_{x \in W} \frac{1}{2}\left|V_{-1}^{1} \cap N(x)\right|+\sum_{x \in W} \frac{1}{2}\left|V_{-1}^{2} \cap N(x)\right| \\
& =n-2\left|V_{-1}\right|+\frac{1}{2}\left|V_{-1}^{1}\right|+\sum_{x \in W} \frac{1}{2}\left|V_{-1}^{2} \cap N(x)\right| \\
& \geq n+\frac{1}{2}\left|V_{-1}^{1}\right|+\left|V_{-1}^{2}\right|-2\left|V_{-1}\right| \\
& \geq n+\frac{1}{2}\left(\left|V_{-1}^{1}\right|+\left|V_{-1}^{2}\right|\right)-2\left|V_{-1}\right| \\
& \geq n-\frac{3}{2}\left|V_{-1}\right| .
\end{aligned}
$$


Now the result follows from Lemma 9.

\section{Special classes of graphs}

In this section, we determine the signed strong Roman domination number of special classes of graphs.

Proposition 11. For $n \geq 3, \gamma_{s s R}\left(K_{1, n}\right)=1$.

Proof. For any $\gamma_{s s R}\left(K_{1, n}\right)$-function $f$, we have $\gamma_{s s R}\left(K_{1, n}\right)=f(N[v]) \geq 1$ where $v$ is the central vertex of $K_{1, n}$. To prove $\gamma_{s s R}\left(K_{1, n}\right) \leq 1$, let $N(v)=\left\{u_{1}, u_{2}, \ldots, u_{n}\right\}$. If $n$ and $\left\lceil\frac{n}{2}\right\rceil$ have different parity, then define $f: V\left(K_{1, n}\right) \rightarrow\left\{-1,1,2, \ldots,\left\lceil\frac{n}{2}\right\rceil+1\right\}$ by $f(v)=\left\lceil\frac{n}{2}\right\rceil, f\left(u_{i}\right)=-1$ for $1 \leq i \leq$ $\left\lceil\frac{n}{2}\right\rceil, f\left(u_{j}\right)=(-1)^{j}$ for $\left\lceil\frac{n}{2}\right\rceil+1 \leq j \leq n-1$ and $f\left(u_{n}\right)=1$. Clearly, $f$ is a signed strong Roman dominating function on $K_{1, n}$ of weight 1 which implies that $\gamma_{s s R}\left(K_{1, n}\right) \leq 1$ in this case.

Let $n$ and $\left\lceil\frac{n}{2}\right\rceil$ have the same parity. Define $f: V\left(K_{1, n}\right) \rightarrow\left\{-1,1,2, \ldots,\left\lceil\frac{n}{2}\right\rceil+1\right\}$ by $f(v)=$ $\left\lceil\frac{n}{2}\right\rceil+1, f\left(u_{i}\right)=-1$ for $1 \leq i \leq\left\lceil\frac{n}{2}\right\rceil, f\left(u_{j}\right)=(-1)^{j}$ for $\left\lceil\frac{n}{2}\right\rceil+1 \leq j \leq n$. Obviously, $f$ is a signed strong Roman dominating function on $K_{1, n}$ of weight

$$
\omega(f)=\left\lceil\frac{n}{2}\right\rceil+1-\left\lceil\frac{n}{2}\right\rceil+\frac{1}{2}\left\lfloor\frac{n}{2}\right\rfloor-\frac{1}{2}\left\lfloor\frac{n}{2}\right\rfloor=1 .
$$

Thus $\gamma_{s s R}\left(K_{1, n}\right)=1$ and the proof is complete.

Proposition 12. For $r \geq s \geq 2$,

$$
\gamma_{s s R}(S(r, s))= \begin{cases}2-\left\lfloor\frac{r}{2}\right\rfloor-\left\lfloor\frac{s}{2}\right\rfloor & \text { if }\left\lfloor\frac{r}{2}\right\rfloor \leq\left\lceil\frac{s}{2}\right\rceil, \\ 1-s & \text { if }\left\lfloor\frac{r}{2}\right\rfloor \geq\left\lceil\frac{s}{2}\right\rceil+1 .\end{cases}
$$

Proof. Let $u$ and $v$ be the central vertices of $S(r, s)$ with $N(u)-\{v\}=\left\{u_{1}, u_{2}, \ldots, u_{r}\right\}$ and $N(v)-$ $\{u\}=\left\{v_{1}, v_{2}, \ldots, v_{s}\right\}$. We consider two cases.

Case 1. $\left\lfloor\frac{r}{2}\right\rfloor \leq\left\lceil\frac{s}{2}\right\rceil$.

Define $f: V \rightarrow\left\{-1,1,2, \ldots,\left\lceil\frac{\Delta}{2}\right\rceil+1\right\}$ by $f\left(u_{i}\right)=f\left(v_{j}\right)=-1$ for $1 \leq i \leq r, 1 \leq j \leq s$ and $f(u)=$ $\left\lceil\frac{r}{2}\right\rceil+1, f(v)=\left\lceil\frac{s}{2}\right\rceil+1$. Obviously, $f$ is an SStRDF on $S(r, s)$ of weight

$$
\omega(f)=\left\lceil\frac{r}{2}\right\rceil+\left\lceil\frac{s}{2}\right\rceil+2-r-s=2-\left\lfloor\frac{r}{2}\right\rfloor-\left\lfloor\frac{s}{2}\right\rfloor .
$$

Hence, $\gamma_{s s R}(S(r, s)) \leq 2-\left\lfloor\frac{r}{2}\right\rfloor-\left\lfloor\frac{s}{2}\right\rfloor$.

Now, we show that $\gamma_{s s R}(S(r, s)) \geq 2-\left\lfloor\frac{r}{2}\right\rfloor-\left\lfloor\frac{s}{2}\right\rfloor$. Let $g$ be a $\gamma_{s s R}(S(r, s))$-function such that $g(u)$ and $g(v)$ are as large as possible. If either $r, s$ have opposite parity or are even, then $\left\lfloor\frac{r+s}{2}\right\rfloor=\left\lfloor\frac{r}{2}\right\rfloor+\left\lfloor\frac{s}{2}\right\rfloor$ and by Proposition 10, we are done. Suppose that $r$ and $s$ are odd. It follows from $\left\lfloor\frac{r}{2}\right\rfloor \leq\left\lceil\frac{s}{2}\right\rceil$ that $r=s+2$. If $g(v)=-1$ (the case $g(u)=-1$ is similar), then 
$g\left(v_{i}\right) \geq 2$ for each $i$, which implies that $\gamma_{s s R}(S(r, s)) \geq 2 s+1>2-\left\lfloor\frac{r}{2}\right\rfloor-\left\lfloor\frac{s}{2}\right\rfloor$. Henceforth, we may suppose that $g(u), g(v) \geq 1$. If $g\left(u_{i}\right) \geq 1$ for some $i$, then $g(u) \leq\left\lfloor\frac{r}{2}\right\rfloor+1$ and the function $h: V \rightarrow\left\{-1,1,2, \ldots,\left\lceil\frac{r+1}{2}\right\rceil+1\right\}$ defined by $h(u)=\left\lceil\frac{r}{2}\right\rceil+1, h\left(u_{i}\right)=-1$ for $1 \leq i \leq r$ and $h(x)=g(x)$ otherwise, is an SStRDF of weight at most $\omega(g)$, a contradiction with the choice of $g$. Hence $g\left(u_{i}\right)=-1$ for each $1 \leq i \leq r$. Similarly, $g\left(v_{i}\right)=-1$ for each $1 \leq i \leq s$. It follows that $g(u)=\left\lceil\frac{r}{2}\right\rceil+1$ and $g(v)=\left\lceil\frac{s}{2}\right\rceil+1$. Thus

$$
\omega(g)=g(u)+g(v)-r-s \geq\left\lceil\frac{r}{2}\right\rceil+1+\left\lceil\frac{s}{2}\right\rceil+1-r-s=2-\left\lfloor\frac{r}{2}\right\rfloor-\left\lfloor\frac{s}{2}\right\rfloor .
$$

Case 2. $\left\lfloor\frac{r}{2}\right\rfloor \geq\left\lceil\frac{s}{2}\right\rceil+1$.

For any $\gamma_{s s R}(S(r, s))$-function $g$, we have $\omega(g)=g(N[u])+\sum_{i=1}^{s} g\left(v_{i}\right) \geq 1-s$. Now define $f$ : $V \rightarrow\left\{-1,1,2, \ldots,\left\lceil\frac{r+1}{2}\right\rceil+1\right\}$ by $f(u)=\left\lceil\frac{r}{2}\right\rceil+1, f(v)=\left\lfloor\frac{r}{2}\right\rfloor$ and $f(x)=-1$ otherwise. Obviously, $f$ is an SStRDF on $S(r, s)$ of weight $\omega(f)=(r+1)-r-s=1-s$. So $\gamma_{s s R}(S(r, s))=1-s$ in this case and the proof is complete.

Proposition 13. For $n \geq 3, \gamma_{s s R}\left(K_{n, n}\right)=4$.

Proof. Let $X=\left\{u_{1}, u_{2}, \ldots, u_{n}\right\}$ and $Y=\left\{v_{1}, v_{2}, \ldots, v_{n}\right\}$ be the partite sets of $K_{n, n}$. If $n$ and $\left\lceil\frac{n}{2}\right\rceil$ have different parity, then define $f: V\left(K_{n, n}\right) \rightarrow\left\{-1,1,2, \ldots,\left\lceil\frac{n}{2}\right\rceil+1\right\}$ by $f\left(v_{1}\right)=f\left(u_{1}\right)=\left\lceil\frac{n}{2}\right\rceil+1$, $f\left(v_{2}\right)=f\left(u_{2}\right)=2, f\left(u_{i}\right)=f\left(v_{i}\right)=-1$ for $3 \leq i \leq\left\lceil\frac{n}{2}\right\rceil+3, f\left(u_{j}\right)=f\left(v_{j}\right)=(-1)^{j}$ for $\left\lceil\frac{n}{2}\right\rceil+4 \leq j \leq$ $n$. If $n$ and $\left\lceil\frac{n}{2}\right\rceil$ have the same parity, then define $f: V\left(K_{n, n}\right) \rightarrow\left\{-1,1,2, \ldots,\left\lceil\frac{n}{2}\right\rceil+1\right\}$ by $f\left(\nu_{1}\right)=$ $f\left(u_{1}\right)=\left\lceil\frac{n}{2}\right\rceil, f\left(v_{2}\right)=f\left(u_{2}\right)=2, f\left(u_{i}\right)=f\left(v_{i}\right)=-1$ for $3 \leq i \leq\left\lceil\frac{n}{2}\right\rceil+2, f\left(u_{j}\right)=f\left(v_{j}\right)=(-1)^{j}$ for $\left\lceil\frac{n}{2}\right\rceil+3 \leq j \leq n$. Obviously, $f$ is a signed strong Roman dominating function on $K_{n, n}$ of weight 4 which implies that $\gamma_{s s R}(G) \leq 4$.

Now we show that $\gamma_{s s R}\left(K_{n, n}\right) \geq 4$. Assume $f$ is a $\gamma_{s s R}\left(K_{n, n}\right)$-function. If there is no vertex with -1 value, then $\gamma_{s s R}\left(K_{n, n}\right) \geq 2 n>4$. Let $V_{-1} \neq \varnothing$. First, let $\left|V_{-1} \cap X\right| \geq 1$ and $\left|V_{-1} \cap Y\right| \geq$ 1 , and let $u_{1}, v_{1} \in V_{-1}$. Then $\sum_{i=1}^{n} f\left(u_{i}\right)=f\left(N\left[v_{1}\right]\right)-f\left(v_{1}\right) \geq 2$ and $\sum_{i=1}^{n} f\left(v_{i}\right)=f\left(N\left[u_{1}\right]\right)-$ $f\left(u_{1}\right) \geq 2$ which implies that $\gamma_{s s R}\left(K_{n, n}\right)=\omega(f)=\sum_{i=1}^{n} f\left(u_{i}\right)+\sum_{i=1}^{n} f\left(v_{i}\right) \geq 4$. Now, let $\mid V_{-1} \cap$ $X \mid=0$ or $\left|V_{-1} \cap Y\right|=0$. Assume, without loss of generality, that $\left|V_{-1} \cap Y\right|=0$. By definition we must have $f\left(v_{i}\right) \geq\left\lceil\frac{\left|V_{-1}\right|}{2}\right\rceil+1$ for some $i$, say $i=1$. It follows that

$$
\gamma_{s s R}\left(K_{n, n}\right)=\omega(f)=f\left(N\left[v_{n}\right]\right)+\sum_{i=1}^{n-1} f\left(v_{i}\right) \geq n+\left\lceil\frac{\left|V_{-1}\right|}{2}\right\rceil \geq 4
$$

This completes the proof.

We remark that the signed Roman domination number and the signed strong Roman domination number of a graph are not comparable. For instance,

$$
\gamma_{s R}(S(2,3))=-1<\gamma_{s s R}(S(2,3))=0,
$$




$$
\gamma_{s R}(S(2,2))=\gamma_{s s R}(S(2,2))=0
$$

and

$$
\gamma_{s R}(S(4,8))=-2>\gamma_{s s R}(S(4,8))=-3
$$

\section{Trees}

In this section, we establish an upper bound on the signed strong Roman domination number of a tree $T$. First we introduce some terminology and notation. A vertex of degree one is called a leaf, and its neighbor is called a stem. If $v$ is a stem, then $L_{v}$ will denote the set of all leaves adjacent to $v$. A stem $v$ is called strong stem if $\left|L_{\nu}\right|>1$ and end-stem if $T-\left(L_{\nu} \cup\{v\}\right)$ is connected. For a vertex $v$ in a rooted tree $T$, let $C(v)$ denote the set of children of $v, D(v)$ denotes the set of descendants of $v$ and $D[v]=D(v) \cup\{v\}$. Also, the depth of $v$, $\operatorname{depth}(v)$, is the largest distance from $v$ to a vertex in $D(v)$. The maximal subtree at $v$ is the subtree of $T$ induced by $D(\nu) \cup\{\nu\}$, and is denoted by $T_{\nu}$.

For a given positive integer $t \geq 2$, a wounded spider is a star $K_{1, t}$ with at most $t-1$ of its edges subdivided once, and a healthy spider is a star $K_{1, t}$ with all of its edges subdivided once. We say spider for both wounded and healthy spiders.

Lemma 14. If $T$ is a spider of order $n \geq 2$, then $\gamma_{s s R}(T) \leq\left\lceil\frac{n}{2}\right\rceil$.

Proof. Let $T$ be a spider with the central vertex $v$. If $T-N[v]=\varnothing$, then $T$ is the star $K_{1, n-1}$ (a wounded spider) and by Proposition 11 we have $\gamma_{s s R}(T) \leq\left\lceil\frac{n}{2}\right\rceil$. Assume that $T-N[\nu] \neq \varnothing$. Let $V(T)=\left\{v, v_{i}, u_{i} \mid 1 \leq i \leq t\right\} \cup L_{v}$ where $N\left(v_{i}\right)=\left\{v, u_{i}\right\}$ for $i \in\{1, \ldots, t\}$. Assume that $L_{v}=$ $\left\{w_{1}, \ldots, w_{s}\right\}$ if $L_{v} \neq \varnothing$. If $\left|L_{v}\right|=0$, then the function $f$ defined by $f(v)=1, f\left(v_{i}\right)=2$ and $f\left(u_{i}\right)=$ -1 for $i \in\{1,2, \ldots, t\}$, is an SStRDF for $T$ of weight $t+1$. Thus $\gamma_{s s R}(T) \leq \omega(f)=t+1=\frac{n+1}{2}=\left\lceil\frac{n}{2}\right\rceil$. If $\left|L_{\nu}\right|=1$, then define $f$ on $V(T)$ by $f\left(u_{i}\right)=f\left(w_{1}\right)=-1, f\left(\nu_{i}\right)=f(\nu)=2$ for $1 \leq i \leq t$. Clearly, $f$ is an SStRDF of $T$ of weight $t+1$ and hence $\gamma_{s s R}(T) \leq \omega(f)=t+1=\frac{n}{2}$. If $\left|L_{v}\right|=2$, then the function $f$ defined by $f(v)=f\left(v_{i}\right)=2$ and $f\left(u_{i}\right)=f\left(w_{j}\right)=-1$ for $i \in\{1,2, \ldots, t\}$ and $j=1,2$, is an SStRDF of $T$ of weight $t+1$ which implies that $\gamma_{s s R}(T) \leq \omega(f)=t+1=\frac{n-1}{2}<\left\lceil\frac{n}{2}\right\rceil$. Let $\left|L_{v}\right| \geq 3$ and let $T^{\prime}$ be the subtree induced by $\{\nu\} \cup\left\{w_{j} \mid 1 \leq j \leq s\right\}$. Obviously, $T^{\prime}$ is a star and so $\gamma_{s s R}\left(T^{\prime}\right)=1$, by Proposition 11. Let $g$ be a $\gamma_{s s R}\left(T^{\prime}\right)$-function and define $f$ on $V(T)$ by $f\left(u_{i}\right)=-1, f\left(v_{i}\right)=2$ for $1 \leq i \leq t$ and $f(x)=g(x)$ otherwise. Clearly, $f$ is an SStRDF of $T$ of weight $t+1$ and so

$$
\gamma_{s s R}(T) \leq \omega(f)=\omega(g)+t \leq 1+t<\left\lceil\frac{n}{2}\right\rceil .
$$

Lemma 15. If $T$ is a spider of order $n \geq 3$ and $v$ is a central vertex of $T$, then every $\gamma_{s s R}(T)$ function assigns a positive value to $v$. Furthermore, $T$ has a $\gamma_{s s R}(T)$-function that assigns positive integer to each end-stem. 
Proof. Let $f$ be a $\gamma_{s s R}(T)$-function. If $T$ is a star, then clearly $f(v) \geq 1$. Assume that $T$ is not a star, $V(T)=\left\{v, v_{i}, u_{i} \mid 1 \leq i \leq t\right\} \cup L_{v}$ is the vertex set of $T$ and $N\left(v_{i}\right)=\left\{v, u_{i}\right\}$ for $i \in\{1, \ldots, t\}$. Suppose that $L_{v}=\left\{w_{1}, \ldots, w_{s}\right\}$ if $L_{v} \neq \varnothing$. Assume, to the contrary, that $f(v)=-1$. By definition we must have $f\left(w_{j}\right)=2$ for each $1 \leq j \leq s$ and $f\left(v_{i}\right)+f\left(u_{i}\right) \geq 2$. This implies that $\gamma_{s s R}(T)=$ $\omega(f)>\left\lceil\frac{n}{2}\right\rceil$, a contradiction with Lemma 14. Thus $f$ assigns a positive value to $v$.

To prove the second part, we note that $f\left(u_{i}\right)+f\left(v_{i}\right)=f\left[u_{i}\right] \geq 1$ for each $1 \leq i \leq t$. Define $g$ on $V(T)$ by $g\left(u_{i}\right)=-1, g\left(v_{i}\right)=2$ for each $1 \leq i \leq t$, and $g(x)=f(x)$ otherwise. Clearly $g$ is an $\gamma_{s s R}(T)$-function with desired property.

Now we present the main result of this section.

Theorem 16. Let $T$ be a tree of order $n \geq 3$. Then $\gamma_{s s R}(T) \leq \frac{2 n}{3}$, with equality if and only if $T=P_{3 t}$ for some positive integer $t$.

Proof. We proceed by induction on $n$. If $\operatorname{diam}(T)=2$, then $T$ is a star and by Proposition 11 we have $\gamma_{s s R}(T)=1 \leq \frac{2 n}{3}$ with equality if and only if $T=P_{3}$. If $\operatorname{diam}(T)=3$, then $T$ is a double star and it follows from Proposition 12 that $\gamma_{s s R}(T)<\frac{2 n}{3}$. If $T$ is a path, then we deduce from Observation 1 and Proposition 2 that $\gamma_{s s R}(T) \leq \frac{2 n}{3}$ with equality if and only if $T=P_{3 t}$ for some positive integer $t$. Hence, the statement is true for all trees of order $n \leq 5$. Assume that $T$ is an arbitrary tree of order $n \geq 6$ and that the statement holds for all trees with smaller order. As above, we may assume that $\operatorname{diam}(T) \geq 4$ and $\Delta(T) \geq 3$. For a subtree $T^{\prime}$ with $n^{\prime}$ vertices, where $n^{\prime} \geq 3$, the induction hypothesis yields an SStRDF $f^{\prime}$ of $T^{\prime}$ with weight at most $\frac{2 n^{\prime}}{3}$. We shall find a subtree $T^{\prime}$ such that adding a bit more weight to $f^{\prime}$ will yield a small enough SStRDF $f$ for $T$.

Let $P=v_{1} v_{2} \ldots v_{k}$ be a diametral path in $T$ chosen to maximize $d_{T}\left(v_{2}\right)$. Also suppose among paths with this property we choose a path such that $\left|L_{v_{3}}\right|$ is as large as possible. Root $T$ at $v_{k}$. We consider the following cases.

Case 1. $d_{T}\left(v_{2}\right) \geq 4$.

Let $T^{\prime}=T-T_{\nu_{2}},\left|V\left(T_{\nu_{2}}\right)\right|=t$ and $f^{\prime}$ be a $\gamma_{s s R}\left(T^{\prime}\right)$-function. Define $f: V(T) \rightarrow\left\{-1,1,2, \ldots,\left\lceil\frac{\Delta}{2}\right\rceil+\right.$ $1\}$ by $f(x)=f^{\prime}(x)$ if $x \in V\left(T^{\prime}\right), f\left(v_{2}\right)=\left\lceil\frac{t}{2}\right\rceil+1, f\left(v_{1}\right)=\left\lfloor\frac{t}{2}\right\rfloor-1$ and $f(x)=-1$ otherwise. Obviously, $f$ is an SStRDF of $T$ and it follows from the induction hypothesis that

$$
\gamma_{s s R}(T) \leq \omega(f)=\omega\left(f^{\prime}\right)+2 \leq \frac{2(n-t)}{3}+2<\frac{2 n}{3} .
$$

Case 2. $d_{T}\left(v_{2}\right)=3$.

Let $L_{v_{2}}=\left\{v_{1}, u\right\}$. By the choice of diametral path, all end-stems adjacent to $v_{3}$ have degree at most 3 . We consider the following subcases. 
Subcase 2.1. $d_{T}\left(v_{3}\right)=2$.

Let $T^{\prime}=T-T_{\nu_{3}}$ and let $f^{\prime}$ be a $\gamma_{s s R}\left(T^{\prime}\right)$-function. If $\left|V\left(T^{\prime}\right)\right|=2$, then $|V(T)|=6$ and it is easy to see that $\gamma_{s s R}(T)=2<\frac{2 n}{3}$. Suppose that $\left|V\left(T^{\prime}\right)\right| \geq 3$. Define $f$ on $V(T)$ by $f(x)=f^{\prime}(x)$ for every $x \in V\left(T^{\prime}\right), f\left(v_{3}\right)=1, f\left(v_{2}\right)=2$ and $f\left(v_{1}\right)=f(u)=-1$. Clearly, $f$ is an SStRDF for $T$ and by the induction hypothesis we have

$$
\gamma_{s s R}(T) \leq \omega(f)=\omega\left(f^{\prime}\right)+1 \leq \frac{2(n-4)}{3}+1<\frac{2 n}{3} .
$$

Subcase 2.2. $d_{T}\left(v_{3}\right) \geq 3$ and $v_{3}$ is adjacent to an end-stem $w$ of degree 3 such that $w \neq v_{2}$. Let $L_{w}=\left\{w_{1}, w_{2}\right\}$ and $T^{\prime}=T-\left\{w, w_{1}, w_{2}, v_{2}, v_{1}, u\right\}$. If $\left|V\left(T^{\prime}\right)\right|=2$, then $|V(T)|=8$ and it is easy to see that $\gamma_{s s R}(T)=1<\frac{2 n}{3}$. Suppose $\left|V\left(T^{\prime}\right)\right| \geq 3$. Define $f$ on $V(T)$ by $f(x)=f^{\prime}(x)$ for every $x \in V\left(T^{\prime}\right)-\left\{v_{3}\right\}, f\left(v_{3}\right)=1$ if $f^{\prime}\left(v_{3}\right)=-1$ and $f\left(v_{3}\right)=f^{\prime}\left(v_{3}\right)$ otherwise, $f\left(v_{2}\right)=f(w)=2$ and $f(x)=-1$ for each $x \in\left\{w_{1}, w_{2}, u, v_{1}\right\}$. Obviously, $f$ is an SStRDF of $T$ and and by the induction hypothesis we obtain

$$
\gamma_{s s R}(T) \leq \omega(f) \leq \omega\left(f^{\prime}\right)+2 \leq \frac{2(n-6)}{3}+2<\frac{2 n}{3} .
$$

By Subcase 2.2, we may assume that all end-stems adjacent to $v_{3}$, with exception $v_{2}$, have degree 2. If $\operatorname{diam}(T)=4$, then $T-T_{\nu_{2}}$ is a spider and applying Lemma 14 yields that $\gamma_{s s R}(T)<\frac{2 n}{3}$. Let $\operatorname{diam}(T) \geq 5$.

Subcase 2.3. $d_{T}\left(v_{3}\right) \geq 3$ and $v_{3}$ is adjacent to an end-stem $w$ of degree 2 .

Let $L_{w}=\left\{w_{1}\right\}$ and $T^{\prime}=T-\left\{v_{1}, v_{2}, u, w, w_{1}\right\}$. Since $\operatorname{diam}(T) \geq 5$, we have $\left|V\left(T^{\prime}\right)\right| \geq 3$. Define $f$ on $V(T)$ by $f(x)=f^{\prime}(x)$ for every $x \in V\left(T^{\prime}\right)-\left\{v_{3}\right\}, f\left(v_{3}\right)=1$ if $f^{\prime}\left(v_{3}\right)=-1$ and $f\left(v_{3}\right)=f^{\prime}\left(v_{3}\right)$ otherwise, $f\left(v_{2}\right)=f(w)=2$ and $f(x)=-1$ for each $x \in\left\{w_{1}, u, v_{1}\right\}$. Obviously, $f$ is an SStRDF of $T$ and by the induction hypothesis we obtain

$$
\gamma_{s s R}(T) \leq \omega(f) \leq \omega\left(f^{\prime}\right)+2+1 \leq \frac{2(n-5)}{3}+3<\frac{2 n}{3} .
$$

Subcase 2.4. $d_{T}\left(v_{3}\right) \geq 3$ and each neighbor of $v_{3}$, with exception $v_{2}, v_{4}$, are leaves.

Let $T^{\prime}=T-T_{\nu_{3}}$. Obviously, $T_{\nu_{3}}$ is a double star and we have $\gamma_{s s R}\left(T_{\nu_{3}}\right) \leq 1$ by Proposition 12 . Let $g$ be a $\gamma_{s s R}\left(T_{\nu_{3}}\right)$-function such that $g\left(\nu_{3}\right) \geq 1$ and define $f$ on $V(T)$ by $f(x)=f^{\prime}(x)$ for every $x \in V\left(T^{\prime}\right)-\left\{v_{4}\right\}, f\left(v_{4}\right)=1$ if $f^{\prime}\left(v_{4}\right)=-1$ and $f\left(v_{4}\right)=f^{\prime}\left(v_{4}\right)$ if $f^{\prime}\left(v_{4}\right) \geq 1$ and $f(x)=g(x)$ otherwise. It is easy to verify that $f$ is an SStRDF of $T$ and by the induction hypothesis we have

$$
\gamma_{s s R}(T) \leq \omega(f) \leq \omega\left(f^{\prime}\right)+2+1 \leq \frac{2(n-5)}{3}+3<\frac{2 n}{3} .
$$

Case 3. $d_{T}\left(v_{2}\right)=2$.

By the choice of the diametral path, we may assume that every end-stem on a diametral path 
has degree 2. In particular, every end-stem adjacent to $v_{3}$ has degree 2 . Thus, it follows that $T_{\nu_{3}}$ is a spider. If $\operatorname{diam}(T)=4$, then $T$ is a spider and by Lemma 14 , we have $\gamma_{s s R}(T) \leq\left\lceil\frac{n}{2}\right\rceil<\frac{2 n}{3}$. Let $\operatorname{diam}(T) \geq 5$. Consider the following subcases.

Subcase 3.1. $d_{T}\left(v_{3}\right) \geq 3$.

Let $T^{\prime}=T-T_{\nu_{3}}$. Since $\operatorname{diam}(T) \geq 5$, we have $\left|V\left(T^{\prime}\right)\right| \geq 3$. Let $g$ be a $\gamma_{s s R}\left(T_{\nu_{3}}\right)$-function such that $g$ assigns a positive value to each end-stem of $T_{\nu_{3}}$ (Lemma 15) and let $\left|V\left(T_{\nu_{3}}\right)\right|=n^{\prime}$. Note that $n^{\prime} \geq 4$. By a closer look at the proof of Lemma 14 , we have $\gamma_{s s R}\left(T_{v_{3}}\right)=\left\lceil\frac{n^{\prime}}{2}\right\rceil$ if and only if $v_{3}$ is adjacent to at most one leaf, and in this case we may assume that $g\left(\nu_{3}\right) \geq 1$ and $g\left(N\left[v_{3}\right]\right) \geq 3$. Define $f$ on $V(T)$ by $f(x)=f^{\prime}(x)$ for every $x \in V\left(T^{\prime}\right)$ and $f(x)=g(x)$ for every $x \in V\left(T_{\nu_{3}}\right)$. It is easy to verify that $f$ is an SStRDF of $T$ and by the induction hypothesis we have

$$
\gamma_{s s R}(T) \leq \omega(f) \leq \omega\left(f^{\prime}\right)+\omega(g) \leq \frac{2\left(n-n^{\prime}\right)}{3}+\frac{n^{\prime}+1}{2} \leq \frac{2 n}{3}-\frac{n^{\prime}-3}{6}<\frac{2 n}{3} .
$$

If $\gamma_{s s R}\left(T_{v_{3}}\right)<\left\lceil\frac{n^{\prime}}{2}\right\rceil$, then define $f$ on $V(T)$ by assigning $f(x)=f^{\prime}(x)$ to every $x \in V\left(T^{\prime}\right), f(x)=$ $g(x)$ to every $x \in V\left(T_{\nu_{3}}\right)-\left\{v_{3}\right\}$ and $f\left(v_{3}\right)=g\left(v_{3}\right)+1$. Again, $f$ is an SStRDF of $T$ and, by the induction hypothesis, we have

$$
\gamma_{s s R}(T) \leq \frac{2\left(n-n^{\prime}\right)}{3}+\frac{n^{\prime}-1}{2}+1<\frac{2 n}{3} .
$$

Subcase 3.2. $d_{T}\left(v_{3}\right)=2$.

By the choice of the diametral path, we may assume that all vertices adjacent to $v_{4}$ with depth 2 , have degree 2 and also, by symmetry, we may assume $d\left(v_{k-1}\right)=d\left(v_{k-2}\right)=2$. Using an argument similar to that described in Case 1, we may assume all end-stems adjacent to $v_{4}$ have degree at most 3.

(a) $d\left(v_{4}\right) \geq 3$ and $v_{4}$ is adjacent to an end-stem of degree 3, say $w$.

Let $w_{1}, w_{2}$ be the leaves adjacent to $w$ and $T^{\prime}=T-\left\{v_{1}, v_{2}, v_{3}, w, w_{1}, w_{2}\right\}$. If $f^{\prime}\left(v_{4}\right)=-1$, then define $f$ on $T$ by $f(x)=f^{\prime}(x)$ for every $x \in V\left(T^{\prime}\right), f\left(w_{2}\right)=1, f(w)=2, f\left(v_{2}\right)=3$ and $f(x)=-1$ for remaining vertices. Also, if $f^{\prime}\left(v_{4}\right) \geq 1$, then define $f$ on $T$ as $f(x)=f^{\prime}(x)$ for every $x \in V\left(T^{\prime}\right), f\left(v_{3}\right)=1, f(w)=f\left(v_{2}\right)=2$ and $f(x)=-1$ for remaining vertices. In both cases, we observe that $f$ is an SStRDF of $T$ and, by the induction hypothesis, we have

$$
\gamma_{s s R}(T) \leq \frac{2(n-6)}{3}+3<\frac{2 n}{3}
$$

(b) $d\left(v_{4}\right) \geq 3$ and $v_{4}$ is adjacent to an end-stem of degree 2, say $w$.

Let $w^{\prime}$ be the leaf adjacent to $w$ and $T^{\prime}=T-\left\{v_{1}, v_{2}, v_{3}, w, w^{\prime}\right\}$. If $f^{\prime}\left(v_{4}\right)=-1$, then define $f$ on $T$ as $f(x)=f^{\prime}(x)$ for every $x \in V\left(T^{\prime}\right), f(w)=f\left(v_{2}\right)=3, f(x)=-1$ for remaining vertices, and if $f^{\prime}\left(v_{4}\right) \geq 1$, then define $f$ on $T$ by $f(x)=f^{\prime}(x)$ for every $x \in V\left(T^{\prime}\right), f(w)=$ 
$f\left(v_{2}\right)=2, f\left(w^{\prime}\right)=f\left(v_{1}\right)=-1$ and $f\left(v_{3}\right)=1$. Clearly, $f$ is an SStRDF of $T$ of weight at most $\omega\left(f^{\prime}\right)+3$ and, by the induction hypothesis, we have

$$
\gamma_{s s R}(T) \leq \frac{2(n-5)}{3}+3<\frac{2 n}{3}
$$

Considering (a) and (b), we may assume that all neighbors of $v_{4}$, with exception $v_{5}$, are leaves or vertices of depth 2. On the other hand, by the choice of diametral path, every neighbor of $v_{4}$ with depth 2 and its neighbor, other than $v_{4}$, have degree 2 .

(c) $d\left(v_{4}\right) \geq 3$ and there is a path $v_{4} w_{3} w_{2} w_{1}$ in $T$ such that $w_{3} \notin\left\{v_{3}, v_{5}\right\}$.

Let $T^{\prime}=T-\left\{v_{i}, w_{i} \mid 1 \leq i \leq 3\right\}$. Define $f$ on $T$ as $f(x)=f^{\prime}(x)$ for every $x \in V\left(T^{\prime}\right), f\left(v_{2}\right)=2$, $f\left(w_{2}\right)=3, f\left(\nu_{3}\right)=1$ and $f\left(\nu_{1}\right)=f\left(w_{1}\right)=f\left(w_{3}\right)=-1$. Obviously, $f$ is an SStRDF of $T$ and

$$
\gamma_{s s R}(T) \leq \frac{2(n-6)}{3}+3<\frac{2 n}{3} .
$$

(d) $d\left(v_{4}\right) \geq 3$ and all neighbors of $v_{4}$, with exception $v_{3}, v_{5}$, are leaves.

Let $L_{v_{4}}=\left\{u_{1}, \ldots, u_{t}\right\}$ and $T^{\prime}=T-T_{v_{4}}$. Define $f$ on $T$ by $f(x)=f^{\prime}(x)$ for every $x \in V\left(T^{\prime}\right)$, $f\left(u_{1}\right)=\left\lfloor\frac{t}{2}\right\rfloor-1, f\left(v_{4}\right)=\left\lceil\frac{t}{2}\right\rceil+1, f\left(v_{3}\right)=1, f\left(v_{2}\right)=2$, and $f(x)=-1$ otherwise. Obviously $f$ is an SStRDF of $T$ of weight $\omega\left(f^{\prime}\right)+3$ and, by the induction hypothesis, we have

$$
\gamma_{s s R}(T) \leq \frac{2(n-(t+4))}{3}+3<\frac{2 n}{3}
$$

(e) $d\left(v_{4}\right)=2$.

First let $d\left(\nu_{5}\right)=2$. Let $T^{\prime}=T-T_{\nu_{5}}$ and let $f^{\prime}$ be a $\gamma_{s s R}\left(T^{\prime}\right)$-function. Since $\Delta(T) \geq 3$, we have $\left|V\left(T^{\prime}\right)\right| \geq 3$. Define $f$ on $T$ by $f(x)=f^{\prime}(x)$ for every $x \in V\left(T^{\prime}\right), f\left(\nu_{5}\right)=f\left(v_{2}\right)=3$, $f\left(v_{4}\right)=f\left(v_{3}\right)=f\left(v_{1}\right)=-1$. Obviously, $f$ is an SStRDF of $T$ of weight $\omega\left(f^{\prime}\right)+3$ and it follows from the induction hypothesis that $\gamma_{s s R}(T) \leq \frac{2(n-5)}{3}+3<\frac{2 n}{3}$.

Now let $d\left(\nu_{5}\right) \geq 3$. If $T-T_{\nu_{4}}$ has a $\gamma_{s s R}$-function $g$ such that $g\left(\nu_{5}\right) \geq 1$, then define $f$ on $T$ by $f(x)=g(x)$ for every $x \in V\left(T-T_{v_{4}}\right), f\left(v_{4}\right)=1, f\left(v_{3}\right)=f\left(\nu_{1}\right)=-1$ and $f\left(v_{2}\right)=3$. Clearly, $f$ is an SStRDF of $T$ of weight $\omega(g)+2$ and by the induction hypothesis we have

$$
\gamma_{s s R}(T) \leq \frac{2(n-4)}{3}+2<\frac{2 n}{3} .
$$

Henceforth, we may assume that all $\gamma_{s s R}\left(T-T_{v_{4}}\right)$-functions assign -1 to $v_{5}$. It follows that $v_{5}$ is not a stem. Let $T_{0}=T-T_{v_{4}}$ and $g$ be a $\gamma_{s s R}\left(T_{0}\right)$-function. If $w$ is a neighbor of $v_{5}$ with depth one and $d_{T}(w) \geq 3$, then obviously $g(w) \geq 1$. If $g\left(w_{1}\right) \geq 1$ for some $w_{1} \in N(w)-$ $\left\{v_{5}\right\}$, then the function $g^{\prime}$ defined on $V\left(T_{0}\right)$ by $g^{\prime}\left(v_{5}\right)=g\left(w_{1}\right), g^{\prime}\left(w_{1}\right)=-1$ and $g^{\prime}(x)=$ $g(x)$ otherwise, is a $\gamma_{s s R}\left(T_{0}\right)$-function that assigns a positive value to $v_{5}$, a contradiction. Otherwise, the function $g_{1}$ on $V\left(T_{0}\right)$ defined by $g_{1}(w)=g(w)-2, g_{1}\left(v_{5}\right)=1$ and $g_{1}(x)=$ $g(x)$ otherwise, is a $\gamma_{s s R}\left(T_{0}\right)$-function that assigns a positive value to $v_{5}$, a contradiction. Thus all neighbors of $v_{5}$ with depth one, have degree 2 . If $z_{3}$ is a neighbor of $v_{5}$ with depth 
two and $\nu_{5} z_{3} z_{2} z_{1}$ is a path in $T$ with $d\left(z_{1}\right)=1$, then by exploiting the above arguments up to Subcase 3.1, we may suppose that $d\left(z_{3}\right)=d\left(z_{2}\right)=2$. Also, if $w_{4}$ is a neighbor of $v_{5}$ with depth three and $v_{5} w_{4} w_{3} w_{2} w_{1}$ is a path in $T$ with $d\left(w_{1}\right)=1$, then by applying the above arguments, we may assume that $d\left(w_{4}\right)=d\left(w_{3}\right)=d\left(w_{2}\right)=2$. Let $v_{5}$ be adjacent to $m_{i}(i=1,2,3)$ vertices of depth $i$ in $T_{\nu_{5}}$. Suppose $v_{5} w_{4}^{i} w_{3}^{i} w_{2}^{i} w_{1}^{i}\left(1 \leq i \leq m_{3}\right)$ are the paths of length 4 in $T_{\nu_{5}}, v_{5} z_{3}^{j} z_{2}^{j} z_{1}^{j}\left(0 \leq j \leq m_{2}\right)$ are the paths of length 3 in $T_{v_{5}}$, and $v_{5} u_{2}^{k} u_{1}^{k}(0 \leq$ $\left.k \leq m_{1}\right)$ are the paths of length 2 in $T_{\nu_{5}}$. Define $g$ on $T_{\nu_{5}}$ by $g\left(v_{5}\right)=1, g\left(w_{4}^{i}\right)=1, g\left(w_{3}^{i}\right)=$ $g\left(w_{1}^{i}\right)=-1, g\left(w_{2}^{i}\right)=3$ for $1 \leq i \leq m_{3}, g\left(z_{3}^{j}\right)=1, g\left(z_{2}^{j}\right)=2, g\left(z_{1}^{j}\right)=-1$ for $0 \leq j \leq m_{2}$, and $g\left(u_{2}^{k}\right)=2, g\left(u_{1}^{k}\right)=-1$ for $0 \leq k \leq m_{1}$. Clearly, $g$ is an SStRDF on $T_{\nu_{5}}$ of weight less than $\frac{2\left|V\left(T_{5}\right)\right|}{3}$. Now let $T^{\prime}=T-T_{\nu_{5}}$ and $f^{\prime}$ be a $\gamma_{s s R}\left(T^{\prime}\right)$-function. Define $f$ on $T$ by $f(x)=$ $f^{\prime}(x)$ for $x \in V\left(T^{\prime}\right)$ and $f(x)=g(x)$ for $x \in V\left(T_{\nu_{5}}\right)$. Clearly $f$ is an SStRDF of $T$ and by the induction hypothesis we have

$$
\gamma_{s s R}(T)=\omega(f)=\omega\left(f^{\prime}\right)+\omega(g)<\frac{2\left(n-\left|V\left(T_{5}\right)\right|\right)}{3}+\frac{2\left|V\left(T_{v_{5}}\right)\right|}{3}=\frac{2 n}{3} .
$$

This completes the proof.

\section{References}

[1] H. Abdollahzadeh Ahangar, M. A. Henning, Y. Zhao, C. Löwenstein and V. Samodivkin, Signed Roman domination in graphs, J. Comb. Optim., 27 (2014), 241-255.

[2] H. Abdollahzadeh Ahangar, J. Amjadi, S.M. Sheikholeslami, L. Volkmann and Y. Zhao, Signed Roman edge domination numbers in graphs, J. Comb. Optim., 31 (2016), 333-316.

[3] E. W. Chambers, B. Kinnersley, N. Prince and D. B. West, Extremal problems for Roman domination, SIAM J. Discrete Math., 23 (2009), 1575-1586.

[4] E. J. Cockayne, P. A. Dreyer Jr., S. M. Hedetniemi and S. T. Hedetniemi, Roman domination in graphs, Discrete Math., 278 (2004), 11-22.

[5] P. Erdos, L. Posá, On the maximal number of disjoint circuits in a graph, Publ. Math. Debrecen, 9 (1962), 3-12.

[6] O. Favaron, H. Karami, R. Khoeilar and S. M. Sheikholeslami, On the Roman domination number of a graph, Discrete Math., 309 (2009), 3447-3451.

[7] S. M. Sheikholeslami and L. Volkmann, Signed Roman domination number in digraphs, J. Comb. Optim., 30 (2015), 456-467.

[8] S. M. Sheikholeslami and L. Volkmann, The Roman k-domatic number of a graph, Acta Math. Sinica, 27 (2011), 1899-1906.

Department of Mathematics, Azarbaijan Shahid Madani University, Tabriz, I.R. Iran.

E-mail: l.sharghi@azaruniv.edu

E-mail: khoeilar@azaruniv.edu

E-mail: s.m.sheikholeslami@azaruniv.edu 